\title{
Lymphoma in 3 related Rottweilers from a single household
}

\author{
R G Lobetti ${ }^{\mathrm{a}}$
}

\begin{abstract}
Over a period of approximately 1 year, 2 sibling Rottweilers and their dam in the same household developed stage IV and stage III lymphoma, respectively. All 3 initially responded to doxorubicin chemotherapy but relapsed after approximately 3 months and were subsequently euthanased. As no obvious environmental trigger could be identified in these dogs, it is speculated that an underlying genetic predisposition could have played a role in the development of lymphoma in these related dogs.
\end{abstract}

Keywords: canine, dog, environment, familial cancer, lymphoma.

Lobetti R G Lymphoma in 3 related Rottweilers from a single household. Journal of the South African Veterinary Association (2009) 80(2): 103-105 (En.). Bryanston Veterinary Hospital, PO Box 67092, Bryanston, 2021 South Africa

\section{INTRODUCTION}

Lymphoma arises from the malignant clonal expansion of lympho-reticular cells.

Both the primary lymphoid organs (bone marrow, thymus) as well as secondary lymphoid structures (lymph nodes, spleen, and respiratory and gut-associated lymphoid tissue) are potential sites of neoplastic transformation. However, because of continuous lymphocyte trafficking, the malignant transformation of lymphocytes can occur virtually anywhere $^{20}$. Lymphoma is reportedly the most common haematopoietic neoplasm affecting the dog, with an incidence approaching $0.1 \%^{2} ; 80 \%$ of lymphomas are seen in 5-11-year-old dogs .

The precise aetiology of lymphoma in the dog has not been identified although several hypotheses have been investigated but none definitely proven. Suggested aetiologies for canine lymphoma include retroviral infection, environmental contamination with phenoxyacetic acid herbicides, magnetic field exposure, chromosomal abnormalities, and immune dysfunction ${ }^{1,3,5,6,17,21}$. A weak to moderate association with the use of herbicides, exposure to strong magnetic fields, or residence in industrial areas has been observed in preliminary epidemiological studies ${ }^{3,5,6,14,24}$. Both somatic and germ line mutations in the tumour suppressor gene p53 have been reported in some cases ${ }^{21}$ and occasional clustering in related dogs has suggested a heritable component in limited instances ${ }^{17}$.

In a prospective study in the Bull mastiff

${ }^{\text {a} B r y a n s t o n ~ V e t e r i n a r y ~ H o s p i t a l, ~ P O ~ B o x ~ 67092, ~ B r y a n-~}$ ston, 2021 South Africa. E-mail: rlobetti@ mweb.co.za Received: December 2008. Accepted: March 2009. it appeared that the incidence rate was higher and that the distribution pattern of the cases displayed a familial pattern ${ }^{12}$. Clustering has been reported in the Rottweiler and Otterhound ${ }^{17}$. In the Rottweiler referred to by Teske $\mathrm{e}^{17}$, both parents and 1 offspring were confirmed to have lymphoma and it was suspected but not demonstrated in 2 of the siblings, whereas lymphoma of the myocardium was found in 3 closely related Otterhounds.

In humans, familial Hodgkin's lymphoma is estimated to represent approximately 1-1.5 \% of all cases of Hodgkin's disease with a 3-fold increased risk of Hodgkin's lymphoma in 1st-degree relatives ${ }^{19}$. It also has been reported that there is a 4-fold increased risk for siblings of young adult cases and no increased risk for siblings of older adult cases ${ }^{4}$. Proposed causes for familial Hodgkin's lymphoma are shared environmental factors, viral disease and genetic determinants ${ }^{19}$.

Treatment of lymphoma in the dog is often rewarding, with the majority of dogs initially responding favourably to chemotherapy. Dogs treated with combination cyclophosphamide, doxorubicin, vincristine and prednisolone protocols generally achieve a complete response rate of $80-90 \%$, with expected median survival times of 1 year ${ }^{8,9}$.

This article describes lymphoma in 2 Rottweiler siblings and their dam, all 3 bitches having lived in the same household and having been exposed to the same environment.

\section{CASE HISTORIES}

\section{Case 1}

A 5-year-old female Rottweiler was referred for evaluation of acute onset generalised peripheral lymphadenomegaly. On clinical examination the dog was bright and alert, rectal temperature was normal, with generalised peripheral lymphadenomegaly and splenomegaly on abdominal palpation. Fine-needle aspirate cytology from the peripheral lymph nodes showed a homogeneous population of large lymphoblastic lymphocytes with large nuclei and moderate amount of cytoplasm. Diffuse hyperechoic splenomegaly and mesenteric lymphadenomegaly were evident on abdominal ultrasonography. Fine-needle aspirate cytology from the spleen and mesenteric lymph nodes showed a homogeneous population of large lymphoblastic lymphocytes similar to those evident on the cytology of the peripheral lymph nodes. Full haematology and serum biochemistry were within normal limits. Serum thymidine kinase (TK) activity was $5.7 \mathrm{U} / \mathrm{l}$ (normal <7). A diagnosis of stage IV lymphoma was made.

Doxorubicin chemotherapy was started and repeated at 3-weekly intervals for a total of 5 treatments. For each treatment the dose used was $30 \mathrm{mg} / \mathrm{m}^{2}$ given intravenously. The dog showed minimal reaction to the chemotherapy and at the completion of the regimen the splenomegaly and lymphadenomegaly had resolved. Serum TK activity 4 weeks after the last chemotherapy was $1.6 \mathrm{U} /$. Three months later the dog developed generalised peripheral lymphadenomegaly, which was diagnosed as a centroblastic lymphoma on histopathology of a lymph node biopsy. Abdominal ultrasonography, full haematology, and serum biochemistry were all within normal limits. The dog was started on a cyclophosphamide, vincristine and prednisolone (COP) protocol, to which there was an initial response but 3 months later, despite still being on the COP chemotherapy, the peripheral lymph nodes enlarged, and were cytologically diagnosed as lymphoma. Owing to the poor prognosis the dog was euthanased.

\section{Case 2}

Two months after initial diagnosis of case 1 , the full sister was referred for evaluation of acute onset generalised peripheral lymphadenomegaly. The only 
pertinent finding on clinical examination was generalised peripheral lymphadenomegaly. Mild splenomegaly with multiple anechoic areas was the only abnormality evident on abdominal ultrasonography. Fine-needle aspirate cytology from the peripheral lymph nodes and spleen showed the presence of a homogeneous population of lymphoblastic lymphocytes with large nuclei and moderate amount of cytoplasm. Full haematology and serum biochemistry were within normal limits. Serum TK activity was 33.7 U/l. A diagnosis of stage IV lymphoma was made.

As with case 1, doxorubicin chemotherapy was similarly initiated, with the dog showing minimal side effects to the chemotherapy and at the completion of the regimen the splenomegaly and lymphadenomegaly had resolved. Serum TK activity 3 weeks after the last chemotherapy was $2.9 \mathrm{U} / \ell$. One month later the dog developed generalised peripheral lymphadenomegaly, which was diagnosed as a centroblastic lymphoma on histopathology of the lymph nodes. Abdominal ultrasonography, full haematology, and serum biochemistry were all within normal limits. She was started on a COP chemotherapy protocol but despite being on chemotherapy showed a relapse within 2 months and was euthanased.

\section{Case 3}

Thirteen and 11 months after the initial diagnoses of cases 1 and 2, respectively their 7-year-old dam was referred for evaluation of acute onset generalised peripheral lymphadenomegaly. A diagnosis of stage III lymphoma was made. Serum TK activity was not measured.

As with the 2 previous cases, 5 treatments of doxorubicin chemotherapy were given. Three months later she developed generalised peripheral lymphadenomegaly, which was again diagnosed as a centroblastic lymphoma on lymph node histopathology. She was then treated with a combination of doxorubicin and L-asparaginase, to which there was minimal response, and was euthanased 4 months later.

\section{DISCUSSION}

Lymphoma is an important and relatively common canine tumour, with an increased breed prevalence reported in one study in the Boxer, Scottish terrier, basset, Airedale terrier, Chow, German shepherd, Poodle, Beagle, Golden retriever, Bulldog and St Bernard ${ }^{22}$, but not in the Rottweiler. In another study, breed predilection for lympho-proliferative diseases was identified and also that certain breeds (Golden retrievers, Labrador retrievers, Cocker spaniels, Rottweilers, Boxers,
German shepherd, and Doberman pinschers) were more likely to develop lymphomas ${ }^{11}$, whereas there was no association with sex and neutering status. It remains, however, unusual that 2 Rottweiler siblings would develop stage IV lymphoma within 3 months of each other, both having a similar response to chemotherapy, and both showing subsequent relapse. In addition, the dam of the 2 siblings developed stage III lymphoma approximately 1 year after the siblings. Possible aetiologies for lymphoma in these Rottweilers would be genetic and/ or environmental factors and/or infectious agents as the dogs were kept in the same household and fed the same food. Approximately 6 months after the lymphoma diagnoses in the 2 siblings the sire, also resident in the same household and exposed to the same food and environment, developed osteosarcoma of the distal radius. Osteosarcoma has no apparent environmental factor or infectious agent trigger but may have a genetic trigger ${ }^{13,15}$. As diet can play a role in the development of tumours in humans, it is possible that the trigger for the lymphoma in these 3 dogs could have been a dietary carcinogen or a lack of essential fatty acids in the diet, possibly due to heat treatment, that might offer protection against environmental carcinogens. However, no one brand of food was exclusively fed to these dogs and there have been, to date, no reports of an increase in the incidence of lymphoma and other tumours associated with commercial dog food.

Although impaired humoral and cellular immunity have been described in dogs with lymphoma ${ }^{18}$, they have not been directly linked to a possible aetiological role. The immune system was not specifically assessed in these 3 Rottweilers but prior to chemotherapy all 3 dogs had normal white cell counts and distribution and normal serum globulins. There was also no report of chronic diseases that could have been associated with an impaired immune system such as pyoderma, respiratory and intestinal tract infections and chronic ehrlichiosis.

Exposure to herbicides has been speculated but not proven to be associated with lymphoma in the $\operatorname{dog}^{18}$. No herbicides were reported to have been used on the property where these Rottweilers were kept. After the initial diagnosis, the owners searched the property for any possible toxin, without finding any. In addition, no other dogs in the immediate area appeared to be affected and to date there has not been any evidence of other dogs or people from the area being diagnosed with lymphoma, or any other tumours. Further evidence against environmental factors $^{3,5,6}$ playing a role was that the dogs did not reside in an industrial area and there were no magnetic fields in the immediate surroundings.

Dogs with lymphoma have been shown to have a 2-180 times higher serum thymidine kinase (TK) activity than normal dogs and dogs with a TK activity $>30 \mathrm{U} / \mathrm{l}$ had significantly shorter survival times $^{23}$. Of the dogs in this report 1 had a normal TK activity, whereas both had an activity of $33.7 \mathrm{U} / \ell$. In itssibling bitches TK activity was within normal range at the completion of the chemotherapy. Serum TK activity was not determined at the time of relapse. Thymidine kinase is a cellular enzyme which is involved in a salvage pathway of DNA synthesis and is activated in the growth phase of the cell cycle. Its activity has been shown to correlate with the proliferative activity of tumour cells. Clinical studies have reported high serum TK concentrations in a variety of neoplasias, the majority being haematological malignancies. Thymidine kinase appears to be a useful, non-invasive marker in lymphoma, where it correlates with clinical staging and provides marked prognostic information on survival ${ }^{10}$. It has also been shown that TK can be used as a powerful objective tumour marker for prognosis and for predicting relapse before recurrence of clinically detectable disease in dogs with lymphoma undergoing chemotherapy ${ }^{23}$.

Single agent lymphoma chemotherapy protocols, except for doxorubicin, have a lower response rate that is not as durable as combination chemotherapy ${ }^{20}$, which was apparent in the responses seen with these 3 dogs. The doxorubicin protocol was used was because of the owner's travelling constraints.

In humans sporadic lymphomas account for the majority of lymphomas; however, familial predisposition alone or in combination with environmental or occupational exposures account for approximately $5 \%$ of cases $^{16}$. The genetic factors that influence the relative risk for lymphoma have been borne out by sibpair, population genetic, and pedigreebased studies ${ }^{16}$. Increased risk of lymphoma in family members suggests an underlying genetic predisposition or defect; it may also result from shared exposure to environmental carcinogens, such as pesticides and herbicides ${ }^{16}$. Genetic studies in people with lymphoma have identified genes at both the HLA class region I and II loci ${ }^{16}$.

In humans familial Hodgkin's lymphoma is estimated to represent approximately $1-1.5 \%$ of all cases of Hodgkin's lymphoma, with environmental factors, viral agents, and genetic determinants having 
been proposed to explain familial aggregation of risk of Hodgkin's lymphoma ${ }^{19}$. Environmental factors and impaired immunity appear to be unlikely in this cohort of related Rottweilers and thus, as in people, it would appear that a genetic origin would be the most likely cause for the lymphoma in these 3 Rottweilers. Further substantiation for a familial link would be that all 3 dogs had a similar remission time and an overall poor prognosis, despite receiving chemotherapy.

\section{REFERENCES}

1. Bertone E R, Synder L A, Moore A S 2002 Environmental tobacco smoke and risk of malignant lymphoma in pet cats. American Journal of Epidemiology 56: 268-273

2. Dorn C R, Taylor D, Schneider R 1970 The epidemiology of canine leukemia and lymphoma. Bibliotheca Haematologica 36: 403-415

3. Gavazza A, Presciuttini S, Barale R, Lubas G, Gugliucci 2001 Association between canine malignant lymphoma, living in industrial areas, and use of chemicals by $\mathrm{dog}$ owners. Journal of Veterinary Internal Medicine 15: 190-195

4. Grufferman S, Cole P, Smith P G 1997 Hodgkin's disease in siblings. New England Journal of Medicine 296: 248-250

5. Hayes H M, Tarone R E, Cantor K P, Jessen C R, McCurnin D M, Richardson R C 1991 Case control study of canine malignant lymphoma: positive association with dog owner's use of 2,4-dichlorophenoxyacetic acid herbicides. Journal of the Natural Cancer Institute 83: 1226-1231

6. Hayes H M, Tarone R E, Cantor K P 1995 On the association between canine malignant lymphoma and opportunity for exposure to 2,4-dichlorophenoxyacetic acid. Environmental Research 70: 119-125

7. Jacobs R M, Messick J M, Valli V E 2002 Tumours of the haemolymphatic system. In Meuten D J (ed.) Tumours in domestic animals (4th edn), Iowa State Press, Iowa: 119-198

8. Keller E T, MacEwen E G, Rosenthal R C, Helfand S C, Fox L E 1993 Evaluation of prognostic factors and sequential combination chemotherapy with doxorubicin for canine lymphoma. Journal of Veterinary Internal Medicine 7: 289-295

9. Khanna C, Lund E M, Redic KA, Hayden D W, Bell F W, Goulland E L, Klausner J S 1998 Randomized controlled trial of doxorubicin versus dactinomycin in a multiagent protocol for treatment of dogs with malignant lymphoma. Journal of the American Veterinary Medical Association 213: 985-990

10. Madewell B R 2004 Serum thymidine kinase activity: an alternative to histologic markers of cellular proliferation in canine lymphoma. Journal of Veterinary Internal Medicine 18: 595-596

11. Modiano J, Breen M, Burnett R C, Parker $\mathrm{H} \mathrm{G}$, Inusah S, Thomas R, Avery P R, Lindblad-Toh K, Ostrander E A, Cutter GC, Avery A C 2005 Distinct B-cell and T-cell lymphoproliferative disease prevalence among dog breeds indicates heritable risk. Cancer Research 65: 5654-5661

12. Onions D E 1984 A prospective survey of familial canine lymphosarcoma. Journal of the National Cancer Institute 72: 909-912

13. Phillips J C, Stephenson B, Hauck M, Dillberger J 2007 Heritability and segregation analysis of osteosarcoma in the Scottish deerhound. Genomics 90: 354-63

14. Reif J S, Lower K S, Ogilvie G K 1995 Residential exposure to magnetic fields and risk of canine lymphoma. American Journal of Epidemiology 141: 352-359

15. Rosenberger J A, Pablo N V, Crawford P C 2007 Prevalence of and intrinsic risk factors for appendicular osteosarcoma in dogs: 179 cases (1996-2005). Journal of the American Veterinary Medical Association 231: 1076-80

16. Siddiqui R, Onel K, Facio F, Offit K 2004 The genetics of familial lymphomas. Current Oncology Reports 6: 380-387

17. Teske E, de Vos J P, Egberink H F, Vos J H 1994 Clustering in canine malignant lymphoma. Veterinary Ouarterly 16: 134-136

18. Teske E 1994 Canine malignant lymphoma: a review and comparison with human non-Hodgkin's lymphosarcoma. Veterinary Quarterly 16: 209-219

19. Thavaraj V, Kumar R, Arya L S 2002 Familial Hodgkin's disease in two siblings. Indian Pediatrics 39: 79-83

20. Vail D M, Young K M 2007 Hematopoietic tumours. In Withrow S J, MacEwen E D (eds) Small animal clinical oncology (4th edn), W B Saunders, Philadelphia: 699-733

21. Veldhoen N, Stewart J, Brown R, Milner J 1998 Mutations of the p53 gene in canine lymphoma and evidence for germ line p53 mutations in the dog. Oncogene 16: 249-255

22. Vonderhaar M A, Morrison W B 2002 Lymphosarcoma. In Morrison W B (ed.) Cancer in dogs and cats: medical and surgical management (2nd edn). Teton New Media, Jackson, Wyoming: 641-670

23. Von Euler H, Einarsson R, Olsson U, Lagerstedt A S, Eriksson S 2004 Serum thymidine kinase activity in dogs with malignant lymphoma: a potent marker for prognosis and monitoring the disease. Journal of Veterinary Medicine 18: 692-702

24. Zahm S H, Blair A 1992 Pesticides and non-Hodgkin's lymphoma. Cancer Research 52: $5485 \mathrm{~s}$ 